Article

\title{
Consumers' Responses to Front-of-Pack Nutrition Labelling: Results from a Sample from The Netherlands
}

\author{
Manon Egnell ${ }^{1, *}{ }^{\oplus}$, Zenobia Talati ${ }^{2}{ }^{\oplus}$, Marion Gombaud ${ }^{1}$, Pilar Galan ${ }^{1}\left(\mathbb{D}\right.$, Serge Hercberg $\left.{ }^{1,3}{ }^{(}\right)$, \\ Simone Pettigrew ${ }^{4,+}$ (D) and Chantal Julia $1,3,+$ \\ 1 Nutritional Epidemiology Research Team (EREN), Sorbonne Paris Cité Epidemiology and Statistics Research \\ Center (CRESS), U1153 Inserm, U1125 Inra, Cnam, Paris 13 University, 93000 Bobigny, France \\ 2 School of Psychology, Curtin University, Kent St, Bentley, WA 6102, Australia \\ 3 Public Health Department, Avicenne Hospital, AP-HP, 93000 Bobigny, France \\ 4 The George Institute for Global Health, Sydney, NSW 2042, Australia \\ * Correspondence: m.egnell@eren.smbh.univ-paris13.fr \\ + These authors contributed equally to this work.
}

Received: 16 July 2019; Accepted: 2 August 2019; Published: 6 August 2019

\begin{abstract}
Front-of-pack labels (FoPLs) are efficient tools for helping consumers identify healthier food products. Although discussions on nutritional labelling are currently ongoing in Europe, few studies have compared the effectiveness of FoPLs in European countries, including the Netherlands. This study aimed to compare five FoPLs among Dutch participants (the Health Star Rating (HSR) system, Multiple Traffic Lights (MTL), Nutri-Score, Reference Intakes (RIs), and Warning symbols) in terms of perception and understanding of the labels and food choices. In 2019, 1032 Dutch consumers were recruited and asked to select one product from among a set of three foods with different nutritional profiles, and then rank the products within the sets according to their nutritional quality. These tasks were performed with no label and then with one of the five FoPLs on the package, depending on the randomization arm. Finally, participants were questioned on their perceptions regarding the label to which they were exposed. Regarding perceptions, all FoPLs were favorably perceived but with only marginal differences between FoPLs. While no significant difference across labels was observed for food choices, the Nutri-Score demonstrated the highest overall performance in helping consumers rank the products according to their nutritional quality.
\end{abstract}

Keywords: nutritional labelling; food choices; comprehension; perception; Dutch consumers; food policies

\section{Introduction}

Front-of-pack labels (FoPLs) have been identified as a promising strategy to help consumers make healthier food choices at the point of purchase [1-3] and encourage manufacturers to improve the nutritional composition of their products [4,5]. Notably, the implementation of FoPLs has been recommended by the World Health Organization as a 'best-buy' measure to help prevent non-communicable diseases [6]. Given their potential to change consumer food choice architecture, by providing readily interpreted nutritional information, the provision of FoPLs has been identified as an effective nudging strategy [7]. However, the multiplicity of existing schemes, potentially in the same market, may increase confusion among consumers [8]. More specifically in the European Union (EU), according to the regulation, FoPLs may only be voluntary, meaning multiple schemes may coexist [9]. In this context, a request for harmonization at the EU level has prompted new discussions by the EU commission since 2018 to modify the existing regulation [10]. Similar political discussions pertaining 
to the objectives and principles of FoPLs have been included within a Codex Alimentarius e-working group, highlighting government interest in this area [11]. Some European countries have already implemented FoPLs as part of national nutrition prevention programs. Examples of these FoPLs include the Green Keyhole in the Nordic countries since the 1980s [12], the Multiple Traffic Lights (MTL) in the United Kingdom since 2004 [13], the Reference Intakes label (RIs) implemented in 2006 following a voluntary initiative from manufacturers [14], and more recently, the Nutri-Score in France since 2017, and then in Belgium and Spain in 2018 [15]. Other FoPLs have been proposed in recent years, including the Evolved Nutrition Label by a consortium of manufacturers [16], the nutritional circles label proposed by the leading association of the German food sector BLL (Bund für Lebensmittelrecht und Lebensmittelkunde), or the battery system proposed by the Italian government. These latter schemes have not been validated by scientific evidence.

Discussions are still on-going in several European countries as to the most efficient FoPL for their population. In the Netherlands, the 'Choices' system was in place between 2006 and 2016. Initially developed by food manufacturers, and then endorsed by the government, this scheme was abandoned following a request from consumers, as it led to confusion as to the ranking of some foods [17]. Recently, the Dutch government announced the possible introduction of a new FoPL in the Netherlands, and noted that further research should be conducted to identify which labels would perform the best for Dutch consumers [18].

Studies investigating consumer responses to different types of FoPLs have explored various dimensions of intrinsic qualities, such as perceptions, understanding, and/or choice. In this context, Grunert et al. proposed a theoretical framework defining the different steps of FoPL use from perception to use in purchasing situations [19]. Although examining each of these elements provides a clearer picture of consumer reactions to different types of FoPLs, the relative contribution of each of these dimensions to help select an effective scheme varies and requires further investigation. Studies investigating perceptions suggest that FoPLs are generally favorably perceived in the population. However, while positive attitudes for a given system are likely to be required for a scheme to be efficient, there may be a discrepancy between consumer preferences and actual performance of the scheme. Indeed, consumers, and especially those with a higher educational level, tend to prefer schemes providing a larger amount of information, although they may not be able to process this information in purchasing situations where decisions are made in very short time frame [20-23]. Objective understanding, defined as the capacity of consumers to understand the information provided by the label in the way that is intended by its designers [19], is usually tested through ranking tasks, in which consumers are exposed to products displaying a FoPL on the pack and are required to rank their relative healthiness compared to a condition with no label. Studies tend to suggest that this type of measure may show a more contrasted performance across FoPLs, thereby providing a better discrimination across different schemes. Studies investigating consumer choices following exposure to FoPLs have shown contrasting results, depending in particular on the type of method that was used (choice task, virtual/experimental supermarket, or in-store study) [24-36]. Globally, the results of these studies suggest that the effect of FoPLs on consumer choices may be of low magnitude, as consumer purchases are guided by a host of influences, of which nutrition may only be one of several drivers, including price and promotion in particular. However, at the population level, such effects would lead to a substantial impact in terms of public health, contributing to the reduction of the nutrition-related disease burden [37].

The aim of the present study was to assess consumer responses to different FoPLs currently implemented in different countries in the world, in a Dutch sample using the methodology of the FOP-ICE study; an international experimental study comparing the effectiveness of various FoPLs in 12 countries [38]. The effectiveness of five front-of-pack nutrition labels corresponding to different types of FoPL formats-Health Star Rating system, Multiple Traffic Lights, Nutri-Score, Reference Intakes, and Warning symbols-was investigated through the three following dimensions: perception, objective understanding, and food choices. 


\section{Materials and Methods}

\subsection{Population Study and Individual Characteristics}

Participants were recruited in the Netherlands by a web panel provider (Pureprofile), applying quotas for sex (50\% women), age (one third in each of the following categories: 18-30 years, 31-50 years, over 51 years), and yearly household income (one third in each of the following categories: low $(<13,962 €)$, medium $(13,962 €-28,135 €)$, and high $(>28,135 €)$ ). In the online questionnaire, individuals were first asked to provide information on socio-demographic, lifestyle, and nutrition-related characteristics, including sex, age, monthly household income, educational level, involvement in grocery shopping, self-estimated diet quality, and self-estimated level of knowledge in nutrition. Individuals were also asked to declare the frequency purchasing the tested food categories (pizzas, cakes, and breakfast cereals, with response options as "always", "often", "sometimes", and "never"). Those who responded "never" to at least two of the three food categories were ineligible to participate.

The protocol of the study (similar to the FOP-ICE study) was approved by the Institutional Review Board of the French Institute for Health and Medical Research (IRB Inserm n ${ }^{\circ} 17-404$ and 17-404 bis) and the Curtin University Human Research Ethics Committee (approval reference: HRE2017-0760). At the beginning of the survey, participants were invited to give their electronic consent.

\subsection{Stimuli and Front-of-Pack Nutrition Labels}

Three food categories (pizzas, cakes, and breakfast cereals) were selected according to two criteria [38]: (1) commonly available in Dutch supermarkets, and (2) contain products with wide variability in nutritional quality. In each food category, a set of three products with distinct nutrient profiles (higher, medium, and lower nutritional quality) was created, allowing a ranking of products according to their nutritional quality. In order to avoid potential bias on product evaluation (e.g., familiarity, habit), mock packages representing a fictional brand ("Stofer") were developed.

Five FoPLs were tested in the present study (Figure 1), including both nutrient-specific and summary schemes. The nutrient-specific labels were: (1) the Multiple Traffic Lights (implemented in the United Kingdom in 2004), indicating the amounts of energy, fat, saturated fat, sugar, and salt, with a color (green, amber, red) depending on the amount; (2) the Reference Intakes, a monochromatic label displaying the amounts of the same nutrients; and (3) the Warning symbol (implemented in Chile in 2016), advising when the level of a given nutrient exceeds what is considered a healthy amount. Summary FoPLs included: (1) the Nutri-Score, a graded scale of five colors from dark green (associated with the letter A) to dark orange (associated with the letter E), characterizing the overall nutritional quality of the food or beverage and (2) the Health Star Rating system (implemented in Australia and New Zealand in 2014), using a graded scale of stars combined with information on nutrient amounts.

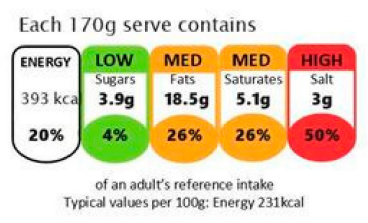

Multiple Traffic Lights

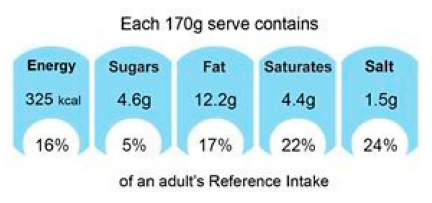

Reference Intakes

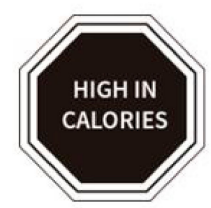

Warning symbol

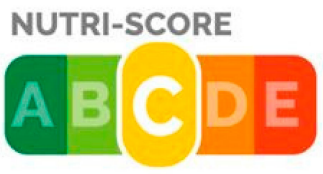

Nutri-Score

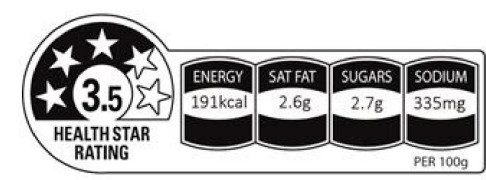

Health Star Rating system

Figure 1. Front-of-pack nutrition labels tested in the present study. 


\subsection{Procedure}

Participants were invited to respond to the online questionnaire that was presented in Dutch. Following the sociodemographic, lifestyle, and nutrition-related questions, participants were asked to complete the choice and understanding tasks, and then answer questions about their perceptions of the FoPL to which they had been assigned.

Given that the first steps of the theoretical framework of FoPL use (perception and understanding) may influence the following step (food choices), the order of the dimensions was reversed in the experiment, starting with choice, followed by understanding and finally perception. First, for each food category, participants were asked to select the product they would be most likely to purchase without any FoPL shown on the mock packages. An "I wouldn't buy any of these products" option was also available. After the choice task, participants were invited to rank the set of three products according to their nutritional quality (1-highest nutritional quality, 2-medium nutritional quality, and 3-lowest nutritional quality), with an "I don't know" option also available and no FoPL on packages. Choice and ranking tasks were completed sequentially for the three food categories. Participants were then randomized to one of the five FoPLs and then invited to fulfill the same tasks, but this time with the assigned FoPL affixed to the mock packages. An example of the choice and ranking tasks for the pizza category is presented in Figure 2.

Participants were then invited to respond to questions about their perceptions on the FoPLs. Various dimensions were assessed including liking (e.g., "I like this label"), awareness (e.g., "this label stands out"), and perceived cognitive workload (e.g., "this label is easy to understand"). For each question, participants provided their responses on a 9-point Likert scale ranging from "strongly disagree" to "strongly agree".

\subsection{Statistical Analyses}

\subsubsection{Food Choice}

For the choice analyses, +1 point was attributed when the lowest nutritional quality product was selected by the participant, +2 points for the medium nutritional quality product and +3 points for the highest nutritional quality product, first for the no labelling condition and then for the FoPL condition. Hence, for each food category, a score was computed using the difference of points between the two conditions, resulting in a discrete continuous score ranging from -2 to +2 points. A global score was finally calculated by summing the score of each category, resulting in a final score between -6 and +6 points. The percentage of participants who deteriorated or improved in their food choices between the no label and FoPL conditions was calculated for each FoPL group by food category. An ordinal logistic regression model was conducted to measure the association between the choice score and FoPL type. Only participants selecting a product in both the no label and FoPL conditions were included in the analyses. 


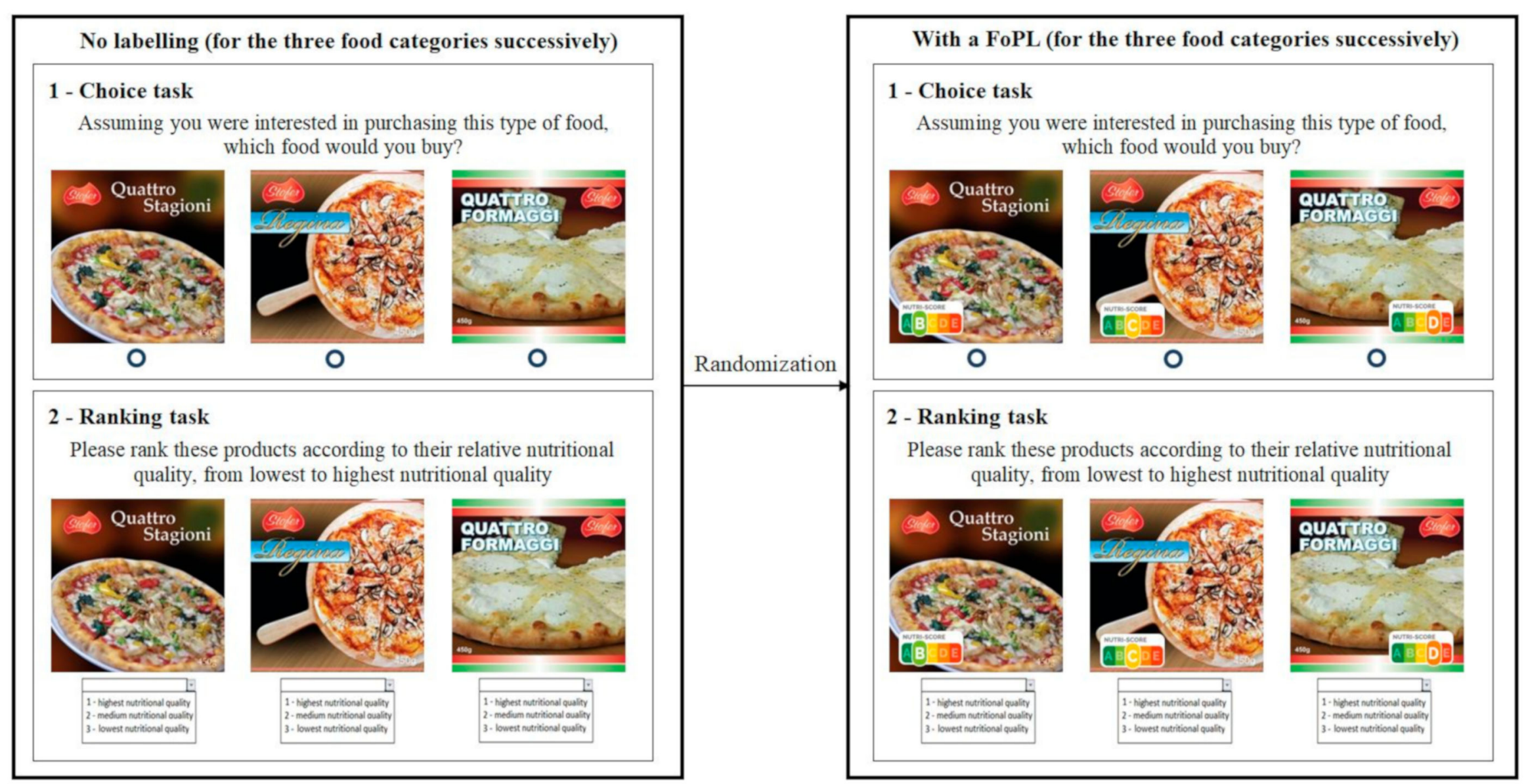

Figure 2. Procedure of the choice and ranking tasks for the pizza category. 


\subsubsection{Objective Understanding}

Objective understanding of the FoPLs by consumers was assessed by the ability of participants to correctly rank the sets of products according to nutritional quality. A response was considered correct when the three products in the set were correctly ranked, leading to a +1 point score for the category. One error (or more) in the ranking task resulted in a -1 point score, while 0 points were attributed when participants selected the "I don't know" answer. Thus, for each food category, a score for ranking ability was calculated using the difference in the number of points between the no label and FoPL conditions, ranging from -2 to +2 points, and leading to a global score of between -6 and +6 points for the three food categories combined. The percentage of correct answers in the no labelling and FoPL conditions was calculated by FoPL type and food category. An ordinal logistic regression model was performed to measure the association between the understanding score and FoPL type.

For the choice and understanding analyses, models were adjusted for individual characteristics including sex, age, level of household monthly income, educational level, involvement in grocery shopping, self-estimated diet quality, and nutrition knowledge, and finally on the response to the question "did you see this label during the survey?". The reference of the models for the FoPL categorical variable was the Reference Intakes label. Interactions between FoPLs and individual characteristics were tested, and stratified analyses were performed when the $p$-value of the interaction term was $\leq 0.10$.

\subsubsection{Perception}

The responses for the assessed perception aspects were characterized for each label by using means and standard deviations. To investigate the contribution of the different questions to the overall perception of FoPLs, principal component analysis was performed. Active variables were "this label is confusing", "I like this label", "this label does not stand out", "this label is easy to understand", "this label takes too long to understand", "this label provides me the information I need", and "I trust this label". Dimensions, corresponding to a linear combination of active variables, have an eigenvalue reflecting the total variance explained by the dimension. The number of retained dimensions was chosen to obtain a cumulative percentage of acceptable variance. In the present study, only the first two dimensions were selected, simplifying the presentation. The contribution and coordinates of each active variable on each axis were computed, indicating how variables contribute to dimensions, and to what extent. The label was considered as a qualitative supplementary variable (not used to compute the dimensions, but mapped on the existing axes). Due to the combination of positive and negative framing of the perception questions, participants who provided the same answers to all perception questions were excluded from the analyses, except those consistently giving a score of five, which indicates a neutral perception.

All analyses in the present study were conducted on SAS statistical software (PROC LOGISTIC, PROC PRINCOMP). Statistical tests were two-sided and a $p$-value $\leq 0.05$ was considered statistically significant.

\section{Results}

\subsection{Description of the Sample}

Individual characteristics of the study sample are described in Table 1 . The present study included 1032 Dutch participants, with $50 \%$ women, $33 \%$ over 51 years, $32 \%$ with a primary or secondary educational level, and $34 \%$ with a low household monthly income. Among all participants, $72 \%$ were responsible for grocery shopping, $11 \%$ had a very or mostly unhealthy diet quality, and $16 \%$ had no or little knowledge about nutrition. 
Table 1. Individual characteristics of the study sample from Netherlands $(\mathrm{N}=1032)$.

\begin{tabular}{|c|c|c|}
\hline & $\mathbf{N}$ & $\%$ \\
\hline \multicolumn{3}{|l|}{ Sex } \\
\hline Men & 517 & 50.1 \\
\hline Women & 515 & 49.9 \\
\hline \multicolumn{3}{|l|}{ Age, years } \\
\hline $18-30$ & 345 & 33.43 \\
\hline $31-50$ & 343 & 33.24 \\
\hline$\geq 51$ & 344 & 33.33 \\
\hline \multicolumn{3}{|l|}{ Educational level } \\
\hline Primary education & 13 & 1.26 \\
\hline Secondary education & 314 & 30.43 \\
\hline Trade certificate & 277 & 26.84 \\
\hline University, undergraduate degree & 329 & 31.88 \\
\hline University postgraduate degree & 99 & 9.59 \\
\hline \multicolumn{3}{|l|}{ Level of household monthly income } \\
\hline High & 342 & 33.14 \\
\hline Medium & 343 & 33.24 \\
\hline Low & 347 & 33.62 \\
\hline \multicolumn{3}{|l|}{ Responsible for grocery shopping } \\
\hline Yes & 746 & 72.29 \\
\hline No & 55 & 5.33 \\
\hline Share job equally & 231 & 22.38 \\
\hline \multicolumn{3}{|l|}{ Self-estimated diet quality } \\
\hline I eat a very unhealthy diet & 8 & 0.78 \\
\hline I eat a mostly unhealthy diet & 102 & 9.88 \\
\hline I eat a mostly healthy diet & 865 & 83.82 \\
\hline I eat a very healthy diet & 57 & 5.52 \\
\hline \multicolumn{3}{|l|}{ Nutrition knowledge } \\
\hline I do not know anything about nutrition & 7 & 0.68 \\
\hline I am not very knowledgeable about nutrition & 157 & 15.21 \\
\hline I am somewhat knowledgeable about nutrition & 744 & 72.09 \\
\hline I am very knowledgeable about nutrition & 124 & 12.02 \\
\hline \multicolumn{3}{|l|}{ Did you see the FOP label during the survey? } \\
\hline No & 293 & 28.39 \\
\hline Unsure & 133 & 12.89 \\
\hline Yes & 606 & 58.72 \\
\hline \multicolumn{3}{|c|}{ Participants who recalled seeing the FoPL they were exposed to } \\
\hline$H S R$ & 111 & 53.62 \\
\hline MTL & 135 & 65.53 \\
\hline Nutri-Score & 147 & 71.36 \\
\hline RIs label & 136 & 53.88 \\
\hline Warning symbol & 77 & 37.20 \\
\hline
\end{tabular}

HSR: Health Star Rating system; MTL: Multiple Traffic Lights; RIs: Reference Intakes.

\subsection{Food Choices}

The percentage of participants who modified their food choices between the no label and FoPL conditions is described in Figure S1. While within each food category and for all five FoPLs, a large number of participants did not change their choice between the two conditions (between $50 \%$ to $63 \%$ depending on the food category and the FoPL), or did not select any product (between $22 \%$ to $41 \%$ depending on the food category and the FoPL), significant modifications in choices occurred in the pizza and cake categories (overall $p$-value for the Bowker disagreement test $=0.0008$ and 0.0001, respectively). Among participants who modified their food choices, a higher percentage demonstrated an improvement in the nutritional quality of their choices (between $2.9 \%$ and $10.7 \%$ depending on the label and the food category) compared to those demonstrating deterioration (between $2.9 \%$ and $5.8 \%$ depending on the label and the food category), with similar results found for the five individual labels. 
Results of the associations between FoPLs and food choices are displayed in Table 2. Compared to the RIs, no significant association was found between FoPLs and the change in nutritional quality of food choices, overall and by food category, except for the Warning symbol. Exposure to the Warning symbol encouraged participants to select a healthier breakfast cereal.

Table 2. Associations between front-of-pack label type and change in nutritional quality of food choices by food category $(\mathrm{N}=1032)$.

\begin{tabular}{|c|c|c|c|c|c|c|c|c|c|}
\hline \multirow{2}{*}{ Food Category } & \multirow{2}{*}{$\mathbf{N}$} & \multicolumn{2}{|l|}{ HSR } & \multicolumn{2}{|l|}{ MTL } & \multicolumn{2}{|l|}{ Nutri-Score } & \multicolumn{2}{|c|}{ Warning Symbol } \\
\hline & & OR $(95 \%$ CI) & $p$ & OR $(95 \%$ CI) & $p$ & OR $(95 \%$ CI) & $p$ & OR $(95 \% \mathrm{CI})$ & $p$ \\
\hline All categories & 898 & $1.21[0.76-1.94]$ & 0.4 & $0.94[0.59-1.51]$ & 0.8 & 1.10 [0.69-1.75] & 0.7 & $1.32[0.82-2.13]$ & 0.3 \\
\hline Pizzas & 692 & $1.11[0.58-2.10]$ & 0.8 & 0.85 [0.45-1.64] & 0.6 & $0.76[0.40-1.44]$ & 0.4 & 0.88 [0.45-1.73] & 0.7 \\
\hline Cakes & 744 & 0.81 [0.44-1.49] & 0.5 & $0.90[0.50-1.63]$ & 0.7 & 1.10 [0.61-1.98] & 0.7 & $0.93[0.50-1.71]$ & 0.8 \\
\hline Breakfast cereals & 643 & $1.72[0.84-3.50]$ & 0.1 & $0.93[0.46-1.88]$ & 0.8 & 1.77 [0.87-3.60] & 0.1 & $2.99[1.45-6.21]$ & 0.003 \\
\hline
\end{tabular}

\subsection{Objective Understanding}

The percentage of correct answers in the nutritional quality ranking task and the improvement between the no label and FoPL conditions are presented (according to FoPL type and food category) in Figure S2. Across all three food categories, the Nutri-Score produced the largest improvement in the percentage of correct answers compared to no label, followed by the MTL. For the other FoPLs, results differed depending on the food category. The associations between FoPL type and the ability to correctly rank products are presented in Table 3, with the RIs label as reference in the models. Overall, the Nutri-Score was the only FoPL to significantly improve participants' ability to correctly rank products according to their nutritional quality compared to the RIs (odds ratio $(\mathrm{OR})=3.60$ [2.48-5.24] $(p$-value $<0.0001))$, while the other FoPLs did not show any significant results. Similar results were found for the three food categories, except for cakes where the Warning symbol (OR = 2.10 [1.32-3.34], $p$-value $=0.002)$ and MTL $(\mathrm{OR}=1.66[1.05-2.62], p$-value $=0.03)$ also significantly improved the ranking ability of participants compared to the RIs, but Nutri-Score remained the label with the highest performance for cakes as well (OR $=4.52$ [2.89-7.06], $p$-value $<0.0001)$.

Table 3. Associations between FoPLs and the ability to correctly rank products according to nutritional quality by food category $(\mathrm{N}=1032)$.

\begin{tabular}{cccccccccc}
\hline \multirow{2}{*}{ Food Category } & \multirow{2}{*}{$\mathbf{N}$} & HSR & & MTL & \multicolumn{2}{c}{ Nutri-Score } & \multicolumn{2}{c}{ Warning Symbol } \\
\cline { 3 - 9 } & & OR (95\% CI) & $p$ & OR (95\% CI) & $p$ & OR (95\% CI) & $p$ & OR (95\% CI) & $p$ \\
\hline All categories & 1032 & $1.20[0.82-1.75]$ & 0.3 & $1.31[0.90-1.90]$ & 0.2 & $3.60[2.48-5.24]$ & $<0.0001$ & $1.23[0.84-1.81]$ & 0.3 \\
Pizzas & 972 & $1.37[0.85-2.21]$ & 0.2 & $1.17[0.73-1.88]$ & 0.5 & $2.12[1.34-3.37]$ & 0.001 & $1.00[0.62-1.62]$ & 1.0 \\
Cakes & 1019 & $1.42[0.89-2.24]$ & 0.1 & $1.66[1.05-2.62]$ & 0.03 & $4.52[2.89-7.06]$ & $<0.0001$ & $2.10[1.32-3.34]$ & 0.002 \\
Breakfast cereals & 931 & $0.90[0.56-1.47]$ & 0.7 & $1.00[0.62-1.62]$ & 1.0 & $2.66[1.68-4.21]$ & $<0.0001$ & $0.85[0.52-1.39]$ & 0.5 \\
\hline
\end{tabular}

The reference of the multivariate ordinal logistic regression for the categorical variable 'label' was the Reference Intakes. The multivariate model was adjusted for sex, age, educational level, level of income, responsibility for grocery shopping, self-estimated diet quality, self-estimated nutrition knowledge level, and "did you see this label during the online survey?" HSR: Health Star Rating system; MTL: Multiple Traffic Lights; OR: Odds Ratio; CI: Confidence Interval. Bold values correspond to significant results ( $p$-value $\leq 0.05$ ).

In sensitivity analyses where respondents who answered "I don't know" were not included, similar trends were observed, though with even higher magnitudes of the effect of FoPLs (Table S1).

No significant interaction with individual characteristics was found, except with sex. However, the interaction was quantitative. 


\subsection{Perception}

The average scores for all perception questions are displayed in Figure S3. Overall, homogeneous results were observed between FoPLs on the various items that were investigated. From principal component analysis, two main dimensions were identified, explaining $44.8 \%$ and $21.1 \%$ of the total variance, respectively. The contribution values and coordinates of active variables on these two dimensions are displayed in Table 4 . The first dimension (horizontal axis) was a linear combination of the responses to the following items: "this label is easy to understand" and "this label provides me the information I need" (which were positively associated with the first dimension), and "this label is confusing" and "this label takes too long to understand" (which were negatively associated with this dimension). The second dimension (vertical axis) was a linear combination of the responses to the following items: "this label takes too long to understand", "this label does not stand out", and "I like this label", which were positively associated with this dimension.

Table 4. Contributions and coordinates of active variables on the two dimensions from the principal component analysis.

\begin{tabular}{lcccc}
\hline \multirow{2}{*}{ Questions } & \multicolumn{2}{c}{ Contributions } & \multicolumn{2}{c}{ Coordinates } \\
\cline { 2 - 5 } & Dimension 1 & Dimension 2 & Dimension 1 & Dimension 2 \\
\hline This label is confusing & 19.59 & 12.88 & -1.65 & 0.92 \\
I like this label & 10.40 & 18.14 & 1.20 & 1.09 \\
This label does not stand out & 7.09 & 20.36 & -0.99 & 1.15 \\
This label is easy to understand & 18.51 & 2.03 & 1.61 & 0.36 \\
This label takes too long to & 15.06 & 22.64 & -1.45 & 1.22 \\
understand & & & & \\
This label provides me the & 16.58 & 13.28 & 1.52 & 0.93 \\
information I need & 12.76 & 10.66 & 1.33 & 0.84 \\
I trust this label & - & - & -0.22 & 0.16 \\
HSR & - & - & 0.38 & 0.44 \\
MTL & - & - & 0.04 & -0.43 \\
Nutri-Score & - & - & -0.05 & 0.32 \\
RIs label & - & - & -0.15 & -0.49 \\
Warning symbol & & & & \\
\hline
\end{tabular}

Labels do not have contribution values given that they were considered as qualitative supplementary variables and were thus not used to compute the dimensions.

When the label was mapped on the two axes as an illustrative variable, the graphic in Figure 3 was obtained. Differences between the FoPLs on the two dimensions appeared to be of very low magnitude (the position on the dimensions was between -0.5 and +0.5 ), although the MTL appeared opposed to the Nutri-Score and the Warning symbol on the second dimension. The MTL therefore appeared to somewhat be the preferred label, but compared to the Nutri-Score and Warning symbol, the MTL took too long to understand and did not stand out. 


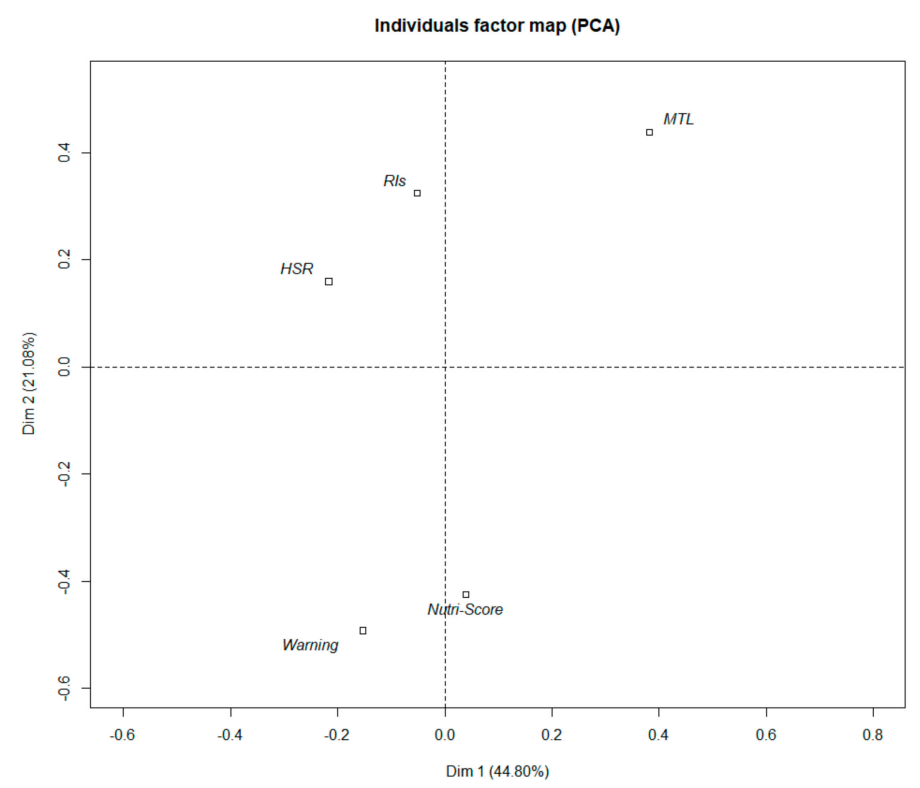

Figure 3. Principal component analysis map showing projection of the labels on the two axes.

\section{Discussion}

While no significant discrimination across FOPLs was observed in terms of perceptions and effect on food choices, the analyses of objective understanding of the labels showed significant differences across schemes. The Nutri-Score demonstrated the highest performance compared to the Reference Intakes in helping Dutch consumers identify and rank the nutritional quality of foods. The other FoPLs did not show any significant effects compared to the RIs except the MTL and Warning symbol for cake products, but to lesser extents. These results, specific to Dutch consumers, are consistent with the findings of the FOP-ICE study, where stronger overall performance of the Nutri-Score was observed for participants' ability to correctly rank the nutritional quality of products in all countries, including the following European countries: Bulgaria, Denmark, France, Germany, Spain, and the United Kingdom [38-40].

The analyses exploring consumers' perceptions of the FoPLs showed that all five FoPLs were favorably perceived. While variations across participants were substantial on the two dimensions of the principal component analysis, the differences by FoPL type were much smaller in magnitude. Moreover, familiarity appeared to influence perceptions, as RIs-that have been implemented as front-of-pack labels on the majority of food products worldwide since 2006-appeared to be appreciated by consumers compared to other labels. Finally, labels providing more accurate information (nutrient-based approaches with numerical information) appeared to be considered somewhat more trustworthy, especially among individuals with higher educational level or substantial knowledge, according to the literature, although they were less salient and entailed a higher cognitive workload [21-23,41]. The limited ability of studying perception to discriminate across labels might be related to the inter-subject approach used in this study (each participant was exposed to one FoPL only), while an intra-subject approach may have yielded more contrasted results (all participants exposed to all FoPLs).

Most previous studies investigating the effects of FoPLs on food choices have focused on the MTL or the RIs and their variants, and have yielded somewhat mixed results. The findings of these studies have typically shown that the RIs have no or limited effect on food choices [35,42-44], whereas the more interpretive MTL can help guide consumers towards healthier foods [26,28,33,35,45,46]. Few studies have investigated more recent schemes, including the Warning symbol, the HSR, and the Nutri-Score, and even fewer in a comparative design, though the results to date in studies using choice sets or experimental design in supermarkets have suggested that these interpretive labels can have a positive effect on the nutritional quality of food choices [30,32,35,42,44,47-49]. A recent study 
observed a significant improvement in the nutritional quality of food choices associated with the use of a warning label, while no results were observed for the other tested labels (MTL, HSR, and Nutri-Score); nevertheless larger sets (20 products) were used compared to our study, allowing capture of the differences for some labels [50]. Results of studies using choice sets, as in our study, appear to be influenced by the categories of products selected [49], as well as the size and types of products within the choice set [24]. When the effects of FoPLs were investigated in studies assessing purchasing outcomes, the Nutri-Score appeared to have a significant impact $[30,32,47,48]$, while results were contrasted for other labels $[27,34,47,51-57]$. The non-significant effects observed on food choice in the present study could be related first to the use of mock packages featuring a fictional brand differing from a real world setting, and second to the type of methodology that was used. Indeed, even if the experimental design allowed control over potential confounding factors and other purchasing determinants, the choice tasks focused on three products from three food categories only, which limits the magnitude of the effects that could be observed compared to studies measuring the overall shopping cart. However, in our case the number of sets and products within the sets had to remain limited given that three dimensions were investigated in the same survey and the questionnaire could not be too long for participants to complete. In addition, choice and ranking tasks were performed on the same sets and included three products only. Indeed, the ranking of products according to nutritional quality had to be similar regardless of the FoPL used, and the higher the number of products within the set, the harder it is to achieve. The balance between the number of products for each task and overall simplicity for participants was carefully considered. Finally, the results could have been impacted by familiarity with and purchasing habits for the food categories used in the study. However, this bias was minimized first by the use of fictional products and a fictional brand, and second by the fact that participants who declared having never purchased one of the food categories were excluded from the analyses on that specific food category.

Our results on consumer understanding confirmed that interpretive systems, and in particular color-coded FoPLs, have greater potential than purely informative systems to improve the capacity of Dutch consumers to correctly rank the nutritional quality of foods. In our study, compared to the RIs, the Nutri-Score outperformed the other FoPLs in improving consumers' ability to correctly rank products according to nutritional quality. These findings are consistent with the results of studies conducted in Uruguay [42,58], Australia [59], and other European countries [38-40,60]. Summary indicators have been demonstrated to be easier to understand by consumers [43,60,61], whereas nutrient-specific labels require greater cognitive workload. Color-coding, using in particular the green/red scale, provides an easy-to-interpret signal, associated with 'stop' and 'go' signals [62], and has been shown to increase attentional capture $[58,63]$. Moreover, from a biological perspective, red and green are immediately discerned and discriminated by the human eye [64]. Thus, a FoPL combining both summary and color-coded features, such as the Nutri-Score, is associated with a better objective understanding by consumers $[38,60,65]$.

Another interesting issue raised by our results is the relative contribution of the different dimensions (and studies thereof) developed to characterize FoPLs and to compare the efficiency of different models. Overall, this study provides useful information on the relative contribution of each type of dimension to policy-makers in the selection of a FoPL. Consumers' perceptions of FoPLs suggest that all types of labels are considered acceptable by consumers, with a limited discrimination across schemes, especially when using an inter-subject approach. Of greater concern is the finding of discrepancies between label preferences and performance, with the Nutri-Score displaying significantly higher performance on objective understanding compared to the other labels, while at the same time being perceived as less reliable by Dutch participants. By contrast, FoPLs considered more trustworthy and useful (RIs in particular), did not significantly improve the ability of participants to correctly rank the nutritional quality of products. This finding suggests that performance studies relying on the testing of consumer understanding may be one of the most important study types, allowing discrimination across label types, and therefore helping policy-makers in decision-making. Finally, 
results on choice suggest that FoPLs may yield limited effects on consumer choices, but that the results are highly dependent on the type of study that is performed, and in particular on the choice set and task consumers are asked to perform. Studies involving experimental conditions mimicking real-life purchases with a high number of choices and high variability in the nutritional quality of the foods offered may provide more contrasted results across labels and would be also one of the most important potential effects of FoPLs to investigate.

Strengths of our study include the participation of a large number of Dutch consumers from various sociodemographic groups, the investigation of multiple dimensions of FoPL effectiveness, and the comparison across multiple types of FoPL schemes using a randomized approach. A potential learning effect was also avoided by using a randomization of the presentation order within the sets and across food categories. Nevertheless, some limitations need to be acknowledged. First, Dutch participants were recruited online using set quotas, rather than attempting to generate a population representative sample, which requires caution regarding the extrapolation of the results. Moreover, although we were able to take into account several aspects of socio-cultural background, we did not include information on ethnicity, while it may affect consumer responses to FoPLs. Second, as participants were blinded to the hypotheses, no information was provided as to the objective or meaning of the FoPL to which they were exposed. While this reduced priming, it may have led to less favorable perceptions of less familiar FoPLs and to an underestimation of the labels' effects. Moreover, participants did not have access to the nutritional composition of the products used in the study, which differs from real-life situations and might have led to fewer correct responses in the no label condition in the understanding task compared to what would occur in real life settings. However, this limitation applied equally to all FoPLs included in the study. Finally, participants were randomized to one FoPL, which led to an inter-subject comparison of the effects of FoPLs. Combining intra- and inter-subject approaches may yield more contrasted results across FoPLs, as shown in earlier studies $[20,22,41,60]$.

To conclude, it is of major importance to investigate various dimensions of effectiveness before implementing a FoPL in a country; however, all dimensions do not necessarily have the ability to discriminate FoPL performance. It is important to note that even if a FoPL is favorably perceived and liked by consumers, it does not guarantee that it will be well understood and used to inform food choices. Thus, before selecting a FoPL, it appears essential to investigate consumers' ability to understand and use various schemes, as this ability constitutes an essential step for a label to be effective in influencing food purchases and consumption. Among the different label types tested in the study, the Nutri-Score appears to be a valid alternative to help Dutch consumers identify and rank the nutritional quality of food products.

Supplementary Materials: The following are available online at http:/www.mdpi.com/2072-6643/11/8/1817/s1, Table S1: Associations between FoPLs and the ability to correctly rank products according to nutritional quality by food category: sensitivity analyses $(\mathrm{N}=1032)$; Figure S1: Percentage of participants that deteriorated or improved their food choices between the two labelling situations, by food category and FoPL; Figure S2: Percentage of correct answers for the ranking tasks, by food category and FoPL; Figure S3: Average scores with standard deviation of perception questions by FoPL.

Author Contributions: M.E. performed data analyses and interpretation, drafted and revised the paper. C.J. and S.P. conceptualized the project in collaboration with S.H. and Z.T., supervised the data analyses and interpretation, participated in the writing, and critically revised the paper for important intellectual content. C.J. is the guarantor. S.H., Z.T., P.G. and M.G. interpreted the data and critically revised the paper for important intellectual content. All authors had full access to all of the data in the study and can take responsibility for the integrity of the data and the accuracy of the data analysis. All authors have read and approved the final manuscript.

Acknowledgments: The authors would like to thank Mark Orange for creating the mock packages, and all researchers and doctoral students who tested the online survey. We also would like to thank Stefanie Vandevijvere for the Dutch translation of the online survey. The present study received funding from Santé Publique France (French Agency for Public Health).

Conflicts of Interest: The authors declare no conflict of interest. 


\section{References}

1. World Health Organization. Global Strategy on Diet, Physical Activity and Health; WHO: Geneva, Switzerland, 2004; pp. 2-8.

2. Organisation for Economic Co-operation and Development. Promoting Sustainable Consumption-Good Practices in OECD Countries; Organisation for Economic Co-operation and Development: Paris, France, 2008.

3. Kleef, E.V.; Dagevos, H. The growing role of front-of-pack nutrition profile labeling: A consumer perspective on key issues and controversies. Crit. Rev. Food Sci. Nutr. 2015, 55, 291-303. [CrossRef] [PubMed]

4. Vyth, E.L.; Steenhuis, I.H.; Roodenburg, A.J.; Brug, J.; Seidell, J.C. Front-of-pack nutrition label stimulates healthier product development: A quantitative analysis. Int. J. Behav. Nutr. Phys. Act. 2010, 7, 65. [CrossRef] [PubMed]

5. Ni Mhurchu, C.; Eyles, H.; Choi, Y.-H. Effects of a Voluntary Front-of-Pack Nutrition Labelling System on Packaged Food Reformulation: The Health Star Rating System in New Zealand. Nutrients 2017, 9, 918. [CrossRef] [PubMed]

6. World Health Organization. NCDs Tackling NCDs; World Health Organization: Geneva, Switzerland, 2017; Available online: http://www.who.int/ncds/management/best-buys/en/ (accessed on 9 July 2019).

7. Scrinis, G.; Parker, C. Front-of-Pack Food Labeling and the Politics of Nutritional Nudges. Law Policy 2016, 38, 234-249. [CrossRef]

8. Draper, A.K.; Adamson, A.J.; Clegg, S.; Malam, S.; Rigg, M.; Duncan, S. Front-of-pack nutrition labelling: Are multiple formats a problem for consumers? Eur. J. Public Health 2013, 23, 517-521. [CrossRef] [PubMed]

9. Europa Summary of EU legislation. Labeling of Foodstuffs. Regulation (EU) No. 1169/2011; European Union: Brussels, Belgium, 2012.

10. Goiana-da-Silva, F.; Cruz-E-Silva, D.; Miraldo, M.; Calhau, C.; Bento, A.; Cruz, D.; Almeida, F.; Darzi, A.; Araújo, F. Front-of-pack labelling policies and the need for guidance. Lancet Public Health 2019, 4, e15. [CrossRef]

11. Thow, A.M.; Jones, A.; Schneider, C.H.; Labonté, R. Global Governance of Front-of-Pack Nutrition Labelling: A Qualitative Analysis. Nutrients 2019, 11, 268. [CrossRef]

12. Asp, N.-G. Bryngelsson Susanne Health claims in the labelling and marketing of food products: The Swedish food sector's Code of Practice in a European perspective. Scand. J. Food Nutr. 2007, 15, 107-126. [CrossRef]

13. Food Standard Agency. Front-of-Pack Traffic Light Signpost Labelling Technical Guidance; Food Standard Agency: Kingsway, UK, 2007; pp. 2-12.

14. Food and Drink Federation. Reference Intakes (Previously Guideline Daily Amounts); Food and Drink Federation: London, UK, 2017.

15. Arrêté du 31 Octobre 2017 Fixant la Forme de Présentation Complémentaire à la Déclaration Nutritionnelle Recommandée par l'Etat en Application des Articles L. 3232-8 et R. 3232-7 du Code de la Santé Publique|Legifrance. Available online: https://www.legifrance.gouv.fr/eli/arrete/2017/10/31/SSAP1730474A/ jo/texte (accessed on 6 June 2018).

16. ENL Taskforce. Promoting Healthier Diets Through Evolved Nutrition Labelling. ENL Taskforce, 2018. Available online: https:/ec.europa.eu/health/sites/health/files/nutrition_physical_activity/docs/ev_20171130_ co03_en.pdf (accessed on 5 August 2019).

17. Choices International Foundation Dutch Choices Logo Gets One Year Extra. Available online: https: //www.choicesprogramme.org/news-updates/news/dutch-choices-logo-gets-one-year-extra (accessed on 9 July 2019).

18. Niamh Michail Dutch Government Mulls Nutrition Logo. Available online: https://www.foodnavigator.com/ Article/2018/04/18/Dutch-government-mulls-nutrition-logo (accessed on 9 July 2019).

19. Grunert, K.G.; Wills, J.M. A review of European research on consumer response to nutrition information on food labels. J. Public Health 2007, 15, 385-399. [CrossRef]

20. Mejean, C.; Macouillard, P.; Peneau, S.; Hercberg, S.; Castetbon, K. Consumer acceptability and understanding of front-of-pack nutrition labels. J. Hum. Nutr. Diet. 2013, 26, 494-503. [CrossRef]

21. Julia, C.; Peneau, S.; Buscail, C.; Gonzalez, R.; Touvier, M.; Hercberg, S.; Kesse-Guyot, E. Perception of different formats of front-of-pack nutrition labels according to sociodemographic, lifestyle and dietary factors in a French population: Cross-sectional study among the NutriNet-Sante cohort participants. BMJ Open 2017, 7, e016108. [CrossRef] [PubMed] 
22. Ducrot, P.; Mejean, C.; Julia, C.; Kesse-Guyot, E.; Touvier, M.; Fezeu, L.; Hercberg, S.; Peneau, S. Effectiveness of Front-Of-Pack Nutrition Labels in French Adults: Results from the NutriNet-Sante Cohort Study. PLoS ONE 2015, 10, e0140898. [CrossRef] [PubMed]

23. Talati, Z.; Pettigrew, S.; Kelly, B.; Ball, K.; Dixon, H.; Shilton, T. Consumers' responses to front-of-pack labels that vary by interpretive content. Appetite 2016, 101, 205-213. [CrossRef] [PubMed]

24. Aschemann-Witzel, J.; Grunert, K.G.; van Trijp, H.C.; Bialkova, S.; Raats, M.M.; Hodgkins, C.; Wasowicz-Kirylo, G.; Koenigstorfer, J. Effects of nutrition label format and product assortment on the healthfulness of food choice. Appetite 2013, 71, 63-74. [CrossRef] [PubMed]

25. Balcombe, K.; Fraser, I.; Falco, S.D. Traffic lights and food choice: A choice experiment examining the relationship between nutritional food labels and price. Food Policy 2010, 35, 211-220. [CrossRef]

26. Borgmeier, I.; Westenhoefer, J. Impact of different food label formats on healthiness evaluation and food choice of consumers: A randomized-controlled study. BMC Public Health 2009, 9, 184. [CrossRef] [PubMed]

27. Carrad, A.M.; Louie, J.C.-Y.; Milosavljevic, M.; Kelly, B.; Flood, V.M. Consumer support for healthy food and drink vending machines in public places. Aust. N. Z. J. Public Health 2015, 39, 355-357. [CrossRef] [PubMed]

28. Cecchini, M.; Warin, L. Impact of food labelling systems on food choices and eating behaviours: A systematic review and meta-analysis of randomized studies. Obes. Rev. 2016, 17, 201-210. [CrossRef] [PubMed]

29. Christoph, M.J.; Ellison, B. A Cross-Sectional Study of the Relationship between Nutrition Label Use and Food Selection, Servings, and Consumption in a University Dining Setting. J. Acad. Nutr. Diet. 2017, 117, 1528-1537. [CrossRef]

30. Ducrot, P.; Julia, C.; Mejean, C.; Kesse-Guyot, E.; Touvier, M.; Fezeu, L.K.; Hercberg, S.; Peneau, S. Impact of Different Front-of-Pack Nutrition Labels on Consumer Purchasing Intentions: A Randomized Controlled Trial. Am. J. Prev. Med. 2016, 50, 627-636. [CrossRef]

31. Gorski Findling, M.T.; Werth, P.M.; Musicus, A.A.; Bragg, M.A.; Graham, D.J.; Elbel, B.; Roberto, C.A. Comparing five front-of-pack nutrition labels' influence on consumers' perceptions and purchase intentions. Prev. Med. 2018, 106, 114-121. [CrossRef] [PubMed]

32. Julia, C.; Blanchet, O.; Mejean, C.; Peneau, S.; Ducrot, P.; Alles, B.; Fezeu, L.K.; Touvier, M.; Kesse-Guyot, E.; Singler, E.; et al. Impact of the front-of-pack 5-colour nutrition label (5-CNL) on the nutritional quality of purchases: An experimental study. Int. J. Behav. Nutr. Phys. Act. 2016, 13, 101. [CrossRef] [PubMed]

33. Maubach, N.; Hoek, J.; Mather, D. Interpretive front-of-pack nutrition labels. Comparing competing recommendations. Appetite 2014, 82, 67-77. [CrossRef] [PubMed]

34. Ni Mhurchu, C.; Volkova, E.; Jiang, Y.; Eyles, H.; Michie, J.; Neal, B.; Blakely, T.; Swinburn, B.; Rayner, M. Effects of interpretive nutrition labels on consumer food purchases: The Starlight randomized controlled trial. Am. J. Clin. Nutr. 2017, 105, 695-704. [CrossRef] [PubMed]

35. Talati, Z.; Norman, R.; Pettigrew, S.; Neal, B.; Kelly, B.; Dixon, H.; Ball, K.; Miller, C.; Shilton, T. The impact of interpretive and reductive front-of-pack labels on food choice and willingness to pay. Int. J. Behav. Nutr. Phys. Act. 2017, 14, 171. [CrossRef] [PubMed]

36. Waterlander, W.E.; Steenhuis, I.H.M.; de Boer, M.R.; Schuit, A.J.; Seidell, J.C. Effects of different discount levels on healthy products coupled with a healthy choice label, special offer label or both: Results from a web-based supermarket experiment. Int. J. Behav. Nutr. Phys. Act. 2013, 10, 59. [CrossRef]

37. Egnell, M.; Crosetto, P.; D’Almeida, T.; Kesse-Guyot, E.; Touvier, M.; Ruffieux, B.; Hercberg, S.; Muller, L.; Julia, C. Modelling the impact of different front-of-package nutrition labels on mortality from non-communicable chronic disease. Int. J. Behav. Nutr. Phys. Act. 2019, 16. [CrossRef]

38. Egnell, M.; Talati, Z.; Hercberg, S.; Pettigrew, S.; Julia, C. Objective Understanding of Front-of-Package Nutrition Labels: An International Comparative Experimental Study across 12 Countries. Nutrients 2018, 10, 1542. [CrossRef]

39. Egnell, M.; Talati, Z.; Pettigrew, S.; Galan, P.; Hercberg, S.; Julia, C. Comparison of front-of-pack labels to help German consumers understand the nutritional quality of food products. Ernährungs Umsch. 2019, 66, 76-84.

40. Galan, P.; Egnell, M.; Salas-Salvadó, J.; Babio, N.; Pettigrew, S.; Hercberg, S.; Julia, C. Understanding of different front-of-package labels by the Spanish population: Results of a comparative study. Endocrinol. Diabetes Nutr. 2019. [CrossRef]

41. Mejean, C.; Macouillard, P.; Peneau, S.; Hercberg, S.; Castetbon, K. Perception of front-of-pack labels according to social characteristics, nutritional knowledge and food purchasing habits. Public Health Nutr. 2013, 16, 392-402. [CrossRef] [PubMed] 
42. Arrúa, A.; Machín, L.; Curutchet, M.R.; Martínez, J.; Antúnez, L.; Alcaire, F.; Giménez, A.; Ares, G. Warnings as a directive front-of-pack nutrition labelling scheme: Comparison with the Guideline Daily Amount and traffic-light systems. Public Health Nutr. 2017, 20, 2308-2317. [CrossRef] [PubMed]

43. Feunekes, G.I.; Gortemaker, I.A.; Willems, A.A.; Lion, R.; van den Kommer, M. Front-of-pack nutrition labelling: Testing effectiveness of different nutrition labelling formats front-of-pack in four European countries. Appetite 2008, 50, 57-70. [CrossRef] [PubMed]

44. Talati, Z.; Pettigrew, S.; Ball, K.; Hughes, C.; Kelly, B.; Neal, B.; Dixon, H. The relative ability of different front-of-pack labels to assist consumers discriminate between healthy, moderately healthy, and unhealthy foods. Food Qual. Prefer. 2017, 59, 109-113. [CrossRef]

45. Kelly, B.; Hughes, C.; Chapman, K.; Louie, J.C.; Dixon, H.; Crawford, J.; King, L.; Daube, M.; Slevin, T. Consumer testing of the acceptability and effectiveness of front-of-pack food labelling systems for the Australian grocery market. Health Promot. Int. 2009, 24, 120-129. [CrossRef] [PubMed]

46. Van Herpen, E.; Hieke, S.; van Trijp, H.C.M. Inferring Product Healthfulness from Nutrition Labelling: The Influence of Reference Points. Appetite 2013, 72, 138-149. [CrossRef]

47. Crosetto, P.; Muller, L.; Ruffieux, B. Réponses des consommateurs à trois systèmes d'étiquetage nutritionnels en face avant. Cah. Nutr. Diététique 2016, 59, 124-131. [CrossRef]

48. Crosetto, P.; Lacroix, A.; Muller, L.; Ruffieux, B. Modification des achats alimentaires en réponse à cinq logos nutritionnels. Cah. Nutr. Diététique 2017, 52, 129-133. [CrossRef]

49. Tórtora, G.; Machín, L.; Ares, G. Influence of nutritional warnings and other label features on consumers' choice: Results from an eye-tracking study. Food Res. Int. 2019, 119, 605-611. [CrossRef]

50. Acton, R.B.; Jones, A.C.; Kirkpatrick, S.I.; Roberto, C.A.; Hammond, D. Taxes and front-of-package labels improve the healthiness of beverage and snack purchases: A randomized experimental marketplace. Int. J. Behav. Nutr. Phys. Act. 2019, 16, 46. [CrossRef]

51. Dodds, P.; Wolfenden, L.; Chapman, K.; Wellard, L.; Hughes, C.; Wiggers, J. The effect of energy and traffic light labelling on parent and child fast food selection: A randomised controlled trial. Appetite 2014, 73, $23-30$. [CrossRef] [PubMed]

52. Hamlin, R.; McNeill, L. Does the Australasian "Health Star Rating" Front of Pack Nutritional Label System Work? Nutrients 2016, 8, 237. [CrossRef] [PubMed]

53. Sacks, G.; Rayner, M.; Swinburn, B. Impact of front-of-pack "traffic-light" nutrition labelling on consumer food purchases in the UK. Health Promot. Int. 2009, 24, 344-352. [CrossRef] [PubMed]

54. Sacks, G.; Tikellis, K.; Millar, L.; Swinburn, B. Impact of "traffic-light" nutrition information on online food purchases in Australia. Aust. N. Z. J. Public Health 2011, 35, 122-126. [CrossRef] [PubMed]

55. Seward, M.W.; Block, J.P.; Chatterjee, A. A Traffic-Light Label Intervention and Dietary Choices in College Cafeterias. Am. J. Public Health 2016, 106, 1808-1814. [CrossRef] [PubMed]

56. Hamlin, R.P.; McNeill, L.S.; Moore, V. The impact of front-of-pack nutrition labels on consumer product evaluation and choice: An experimental study. Public Health Nutr. 2015, 18, 2126-2134. [CrossRef]

57. Thorndike, A.N.; Riis, J.; Sonnenberg, L.M.; Levy, D.E. Traffic-light labels and choice architecture: Promoting healthy food choices. Am. J. Prev. Med. 2014, 46, 143-149. [CrossRef]

58. Antúnez, L.; Giménez, A.; Maiche, A.; Ares, G. Influence of Interpretation Aids on Attentional Capture, Visual Processing, and Understanding of Front-of-Package Nutrition Labels. J. Nutr. Educ. Behav. 2015, 47, 292-299.e1.

59. Carter, O.; Mills, B.; Phan, T. An independent assessment of the Australian food industry's Daily Intake Guide "Energy Alone" label. Health Promot. J. Aust. 2011, 22, 63-67. [CrossRef]

60. Ducrot, P.; Mejean, C.; Julia, C.; Kesse-Guyot, E.; Touvier, M.; Fezeu, L.K.; Hercberg, S.; Peneau, S. Objective Understanding of Front-of-Package Nutrition Labels among Nutritionally At-Risk Individuals. Nutrients 2015, 7, 7106-7125. [CrossRef]

61. Hersey, J.C.; Wohlgenant, K.C.; Arsenault, J.E.; Kosa, K.M.; Muth, M.K. Effects of front-of-package and shelf nutrition labeling systems on consumers. Nutr. Rev. 2013, 71, 1-14. [CrossRef] [PubMed]

62. Vasiljevic, M.; Pechey, R.; Marteau, T.M. Making food labels social: The impact of colour of nutritional labels and injunctive norms on perceptions and choice of snack foods. Appetite 2015, 91, 56-63. [CrossRef] [PubMed] 
63. Bialkova, S.; Grunert, K.G.; Juhl, H.J.; Wasowicz-Kirylo, G.; Stysko-Kunkowska, M.; van Trijp, H.C.M. Attention mediates the effect of nutrition label information on consumers' choice. Evidence from a choice experiment involving eye-tracking. Appetite 2014, 76, 66-75. [CrossRef] [PubMed]

64. Nagle, M.G.; Osorio, D. The tuning of human photopigments may minimize red-green chromatic signals in natural conditions. Proc. Biol. Sci. 1993, 252, 209-213. [PubMed]

65. Egnell, M.; Ducrot, P.; Touvier, M.; Allès, B.; Hercberg, S.; Kesse-Guyot, E.; Julia, C. Objective understanding of Nutri-Score Front-Of-Package nutrition label according to individual characteristics of subjects: Comparisons with other format labels. PLoS ONE 2018, 13, e0202095. [CrossRef]

(C) 2019 by the authors. Licensee MDPI, Basel, Switzerland. This article is an open access article distributed under the terms and conditions of the Creative Commons Attribution (CC BY) license (http://creativecommons.org/licenses/by/4.0/). 